\title{
Future opportunities in preventing ototoxicity: Caffeic acid phenethyl ester may be a candidate (Review)
}

\author{
SUMEYYA AKYOL $^{1}$, BUNYAMIN ISIK $^{2}$, AYNUR ALTUNTAS $^{3}$, GONUL ERDEN $^{4}$,

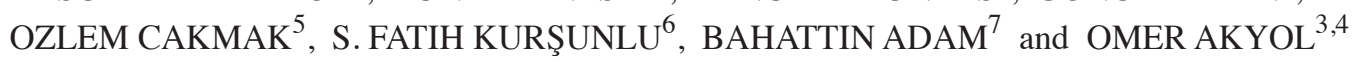 \\ ${ }^{1}$ Department of Medical Biology, Medical Faculty, Turgut Ozal University, Ankara 06170; ${ }^{2}$ Department of \\ Family Medicine, Medical Faculty, Hacettepe University, Ankara 06100; ${ }^{3}$ Division of Chemistry, Ankara Regional Office \\ of Council of Forensic Medicine, Ankara 06300; ${ }^{4}$ Department of Medical Biochemistry, Medical Faculty, \\ Hacettepe University, Ankara 06100; ${ }^{5}$ Department of Biology, Faculty of Education, Gazi University, \\ Ankara 06500; ${ }^{6}$ Department of Periodontology, Faculty of Dentistry, Adnan Menderes University, \\ Aydin 09100, Turkey; ${ }^{7}$ San Jose State University, San Jose 95101, CA, USA
}

Received July 25, 2014; Accepted April 10, 2015

DOI: $10.3892 / \mathrm{mmr} .2015 .3785$

\begin{abstract}
Caffeic acid phenethyl ester (CAPE) is an important active component of propolis, which is derived from honeybee hives. It has received increasing attention in a variety of medical and pharmaceutical research, due to its anti-oxidant, antiproliferative, anti-inflammatory, antiviral and antifungal activity, in addition to its antineoplastic properties. Besides the use of CAPE as an antioxidant and anti-inflammatory agent in a number of in vivo studies of ear disease, its beneficial effects have been reported in the treatment of cancer, arthritis, allergies, heart disease, diabetes, kidney disease, liver disease and neurological disease. CAPE influences a number of biochemical pathways, as well as several targets involved in ear diseases, in particular, in ototoxicity. The protective effects of CAPE in ototoxicity, which may be induced by a number factors, including lipopolysaccharides, hydrogen peroxide and streptomycin, are evaluated and discussed in the present review.
\end{abstract}

\section{Contents}

1. Introduction

2. Use of CAPE in antibiotic-induced ototoxicity

3. Use of CAPE in otitis media

4. Use of CAPE in hydrogen peroxide-induced oxidative stress in HMEEC cells

Correspondence to: Dr Sumeyya Akyol, Department of Medical Biology, Turgut Ozal University Medical Faculty, Anadolu Bulvari No: 16/A, Ankara 06170, Turkey

E-mail: sumeyyaak@hotmail.com; sumeyyaak@gmail.com

Key words: caffeic acid phenethyl ester, ear, ototoxicity, protection, antioxidant
5. Effect of CAPE in myringosclerosis

6. Conclusions

\section{Introduction}

Caffeic acid phenethyl ester (CAPE; Fig. 1), a well known component of the natural honeybee product, propolis, has been used for centuries in medicine, due to its anti-inflammatory, antioxidant and antineoplastic properties (1-4). CAPE is a naturally occurring phenolic compound, and is an ester derived from caffeic acid and phenethyl alcohol. It downregulates a number of pro-inflammatory cytokines and inflammatory mediators, by inhibiting the transcription of nuclear factor $\kappa$-light-chain-enhancer of activated $B$ cells $(\mathrm{NF}-\kappa \mathrm{B})(5)$. CAPE exhibits antimitogenic, anticarcinogenic, anti-inflammatory and immunomodulatory properties in vitro (6). There are a number of articles that have reviewed the positive effects of CAPE in models of neoplasm (7), prostate, lung and melanoma cancers (8), chemotherapy and radiotherapy-induced toxicity (9), and in heart disease (10). However, to the best of our knowledge, there has been no review discussing the protective role of CAPE on diseases of the ear, such as ototoxicity.

Reactive oxygen species (ROS) are associated with ototoxicity and presbycusis (11). Not all cell types found in the cochlea share the same vulnerability to ROS injury. Outer hair cells on the base of the cochlea are susceptible to ROS, while supporting cells show significantly greater survival capacity compared with hair cells, following exposure to ROS (12). Glutathione levels were found to be higher in apical outer hair cells, compared with outer hairs at the base (12). The literature demonstrates that oxidative stress, regardless of its origin, is associated with ototoxicity (13).

Since a number of oxidative pathways may affect the structures in the ear (Fig. 2), CAPE may be considered as a promising agent with which to prevent ROS-induced ototoxicity caused by certain medicines, myringosclerosis, otitis media and inflammation of the ear. Therefore, the present 
review aims to summarize and critically evaluate the evidence for the protective role of CAPE in ototoxicity.

\section{Use of CAPE in antibiotic-induced ototoxicity}

Aminoglycoside antibiotics, which are polycationic compounds, are widely utilized in clinical practice. However, they induce adverse ototoxic effects in $2-5 \%$ of patients (14). The common cochleotoxic or vestibulotoxic side effects thus limit the use of aminoglycoside antibiotics. A number of the oxidation products of polyunsaturated fatty acids (PUFA) in phospholipids act as mediators of apoptosis. ROS activate apoptotic or necrotic intracellular pathways, including the c-Jun N-terminal kinase (JNK) pathway (15). The inhibition of the JNK pathway has been demonstrated to help hair cells injured by aminoglycosides (15). Due to the fact that the trigger mechanism starts with ROS activation, CAPE may be a promising agent to block this cascade at two different points (Fig. 3). A number of studies have investigated underlying mechanisms of action of numerous antibiotics and cytotoxic medications, which result in ototoxicity (16-18). Once inside the negatively charged apex of the hair cell, aminoglycosides induce the generation of ROS, which are involved in molecular pathways leading to ototoxicity (19). Bakir et al (20) conducted a study in order to assess the antioxidant properties of CAPE in the prevention or attenuation of ototoxicity caused by long-term use of aminoglycosides in a rat model. Specifically, the authors investigated the toxic effects of intramuscular injections of streptomycin (20 mg/kg/day for 45 days). On day 45, a marked decrease in cochlear activity was observed in the streptomycin group compared with that in the control group, according to distortion product otoacoustic emissions (DPOAEs). However, no significant differences were observed between the control and CAPE groups. The distortion product-gram, DP-gram (a measurement for DPOAEs) of the streptomycin group had significantly deteriorated compared with the control and streptomycin plus CAPE-treated rats. The number of cochlear hair cells was shown to be reduced in rats treated with streptomycin. Immunohistochemical examination revealed caspase-3 immunoreactivity in rats treated with streptomycin, while this was not observed in streptomycin plus CAPE-treated rats, which indicates a protective effect of CAPE on hair cells, supporting cells, and the basilar membrane. CAPE, when applied alone, exhibited no obvious harmful effects on hair cells. Histopathological examination with hematoxylin and eosin, and immunohistochemistry, in addition to hearing test measurements, did not reveal deterioration in the cochlear hair cells of rats in the CAPE-treated group. These results demonstrate that CAPE exhibits protective effects against ROS generated by oxidative pathways in the ear (Fig. 2).

More recently, Sakallioglu et al (21) examined the effects of exogenous glucocorticoid exposure during the prenatal effect on hearing, in addition to the protection of this inner ear injury by CAPE. Dexamethasone, which is capable of causing an excessive production of ROS, was administered to pregnant Sprague-Dawley rats (one group received only dexamethasone, and the other received dexamethasone plus CAPE) prior to exposure to $110 \mathrm{~dB}$ of noise for $4 \mathrm{~h}$. Exogenous dexamethasone administration did not alter hearing thresholds prior to the noise exposure. Following noise exposure, dexamethasone

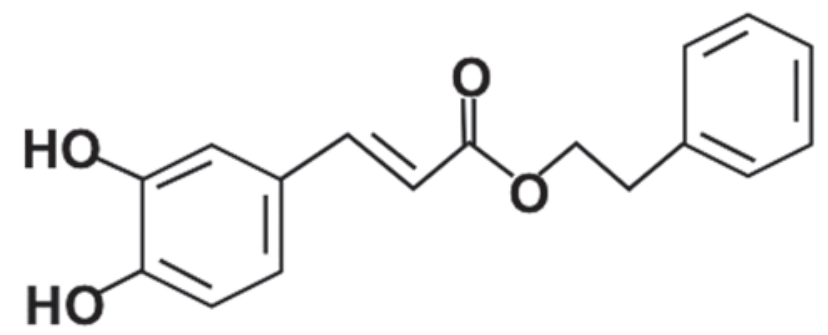

Figure 1. Chemical structure of caffeic acid phenethyl ester.

treatment elevated the hearing thresholds. Therefore, prenatal exposure to dexamethasone may cause inner ear susceptibility to noise. In this study, CAPE treatment did not prevent the damage to hearing caused by dexamethasone. This finding supports the hypothesis that the protective effects of CAPE are due to its antioxidant and anti-inflammatory properties. In conclusion, CAPE does not appear protect the inner ear against dexamethasone-induced oxidative stress.

\section{Use of CAPE in otitis media}

Otitis media (OM) is the leading cause of visits to physicians and consequent antibiotic prescriptions, and is a common cause of hearing impairment in children (22). Pro-inflammatory mediators have been reported to be responsible for the inflammation observed in OM. Several cytokines, including fibroblast growth factor, tumor necrosis factor $\alpha$ (TNF- $\alpha)$, platelet activating factor, interleukin (IL) $2,6,8$ and $1 \beta$, and interferon $\gamma$, have been isolated in middle ear effusions, and are responsible for increases in vascular permeability, chemotaxis, secretion, and consequently, middle ear inflammation (Fig. 4) (23). Additionally, lipopolysaccharide (LPS) is a pro-inflammatory mediator that is associated with middle ear inflammation. Furthermore, a number of other ROS products may directly damage the epithelium of the middle ear. For example, nitric oxide (NO) has been reported to contribute to vasodilatation, increased vascular permeability and mucoid effusions (24). Furthermore, NO produced by activated inflammatory cells, regulates cells and induces inflammation. Therefore, NO may be a secondary inflammatory mediator, produced by the middle ear epithelium in response to primary cytokines, such as IL-1 $\beta$, TNF- $\alpha$ (25).

Previously, in vivo and in vitro studies have shown that cytokines increase inducible nitric oxide synthase (iNOS) production in middle ear epithelial cells (26). Intracellular NO production inhibition prevents the hypersecretion of mucin that is stimulated by ROS and other inflammatory mediators (27). A number of studies have suggested that CAPE is involved in the suppression of LPS-induced inflammatory responses in the HMEEC human middle ear epithelial cell line, via the inhibition pro-inflammatory mediators (Fig. 4) (28). Thus, the effects of CAPE on TNF- $\alpha$, may underlie the LPS-stimulation of HMEECs. In these studies, the bacterial endotoxin, LPS $(10 \mu \mathrm{g} / \mathrm{ml})$, which is isolated from Pseudomonas aeruginosa, was used in order to induce an inflammatory response in HMEEC cells. CAPE was administered at doses of $0,10,50$, 100 and $200 \mu \mathrm{M}$ for $1 \mathrm{~h}$. LPS treatment led to an increase in TNF- $\alpha$ gene expression in HMEEC cells. CAPE treatment 

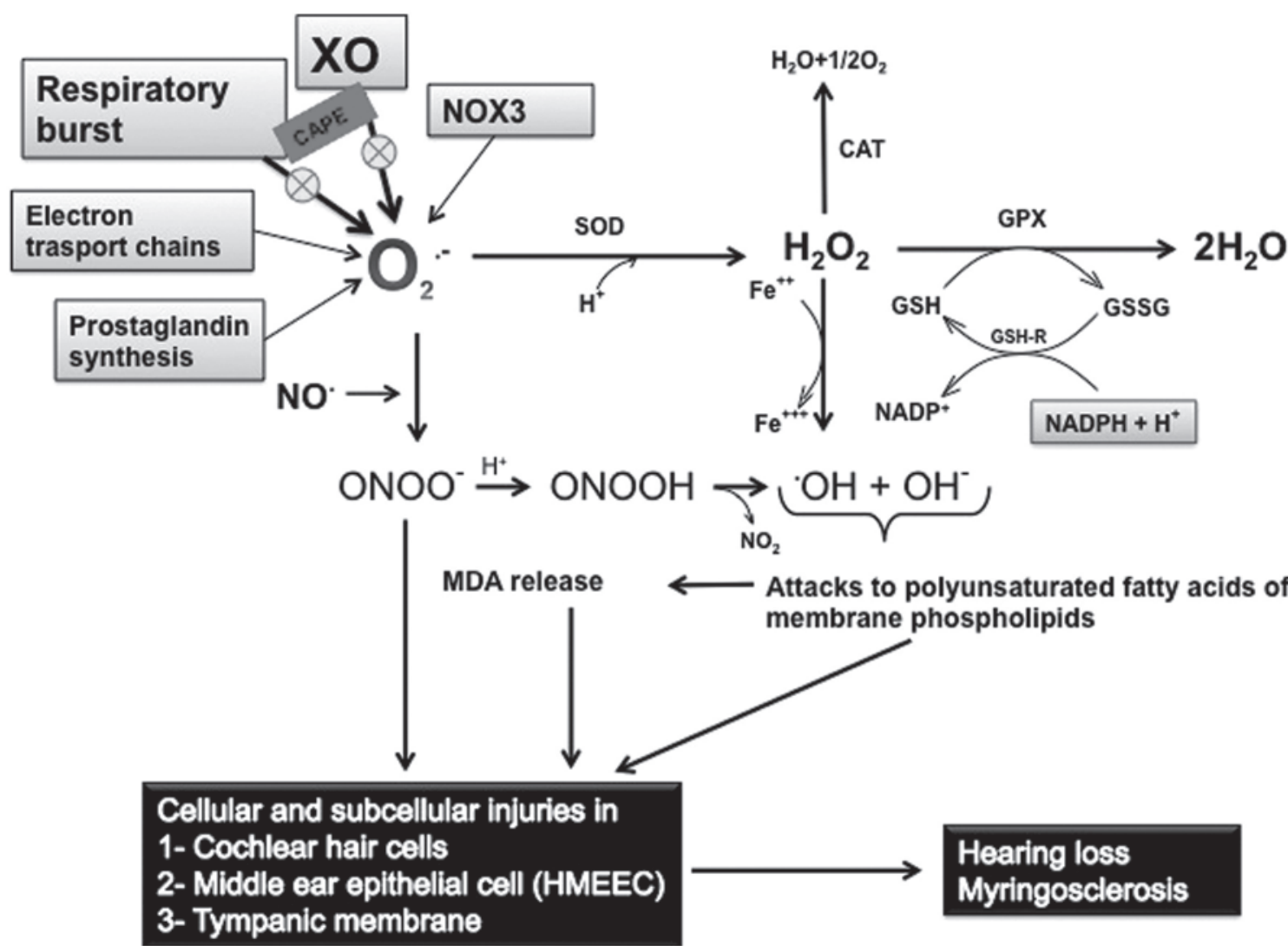

Figure 2. Proposed mechanism for the induction of oxidative stress, and its effects on hearing pathways and cells, as well as the use of CAPE for mitigating the damaging effects of ROS. CAT, catalase; $\mathrm{Fe}^{++}$, ferrous iron; GPx, glutathione peroxidase; GSH, reduced glutathione; GSH-Red, glutathione reductase; GSSG, oxidized glutathione; $\mathrm{H}^{+}$, hydrogen ion proton; $\mathrm{H}_{2} \mathrm{O}$, water; $\mathrm{H}_{2} \mathrm{O}_{2}$, hydrogen peroxide; MDA, malondialdehyde; $\mathrm{NADP}^{+}$, oxidized nicotinamide adenine dinucleotide phosphate; $\mathrm{NADPH}+\mathrm{H}^{+}$, reduced nicotinamide adenine dinucleotide phosphate; $\mathrm{NO}$, nitric oxide radical; $\mathrm{NO}_{2}$, nitrite; $\mathrm{NOX} 3$, $\mathrm{NADPH}$ oxidase 3; $\mathrm{O}_{2}$, molecular oxygen; $\mathrm{O}_{2}^{-}$, superoxide anion radical; $\mathrm{OH}^{-}$, hydroxyl ion; $\mathrm{OH}$, hydroxyl radical; $\mathrm{ONOO}^{-}$, peroxynitrite; PUFA, polyunsaturated fatty acid; $\mathrm{SOD}$, superoxide dismutase; tNOS, total nitric oxide synthases; XO, xanthine oxidase; ROS, reactive oxygen species; CAPE, caffeic acid phenethyl ester.

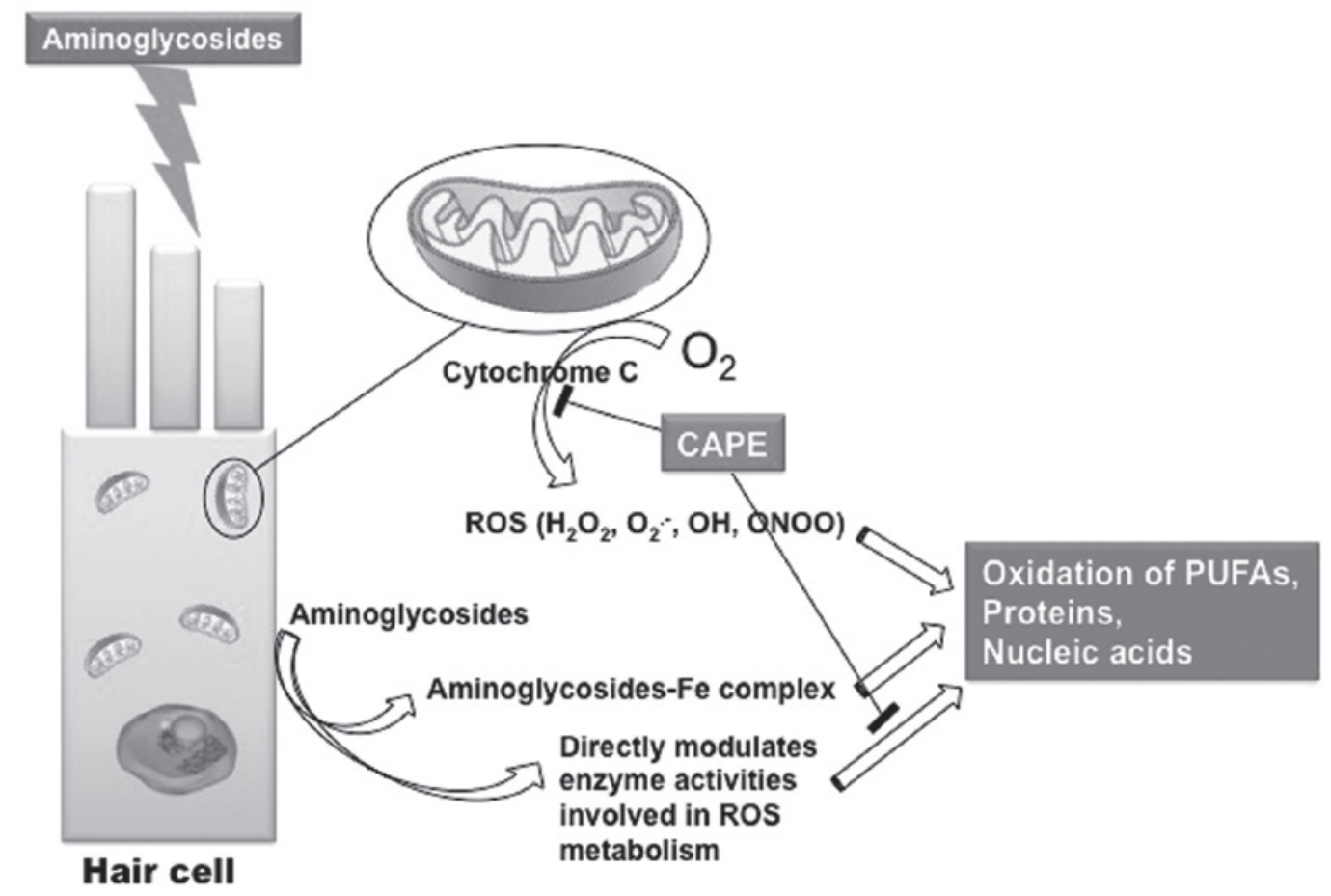

Figure 3. Schematic representation of the ototoxic effects of aminoglycosides. A number of the oxidation products of PUFA in phospholipids, which are located particularly in membranous structures, act as mediators of apoptosis. $\mathrm{H}_{2} \mathrm{O}_{2}$, hydrogen peroxide; $\mathrm{O}_{2}$, molecular oxygen; $\mathrm{O}_{2}^{-}$, superoxide radical; $\mathrm{OH}$, hydroxyl radical; ONOO, peroxynitrite; ROS, reactive oxygen species; PUFA, polyunsaturated fatty acids; ROS, reactive oxygen species; CAPE, caffeic acid phenethyl ester; JNK, c-Jun N-terminal kinase. 


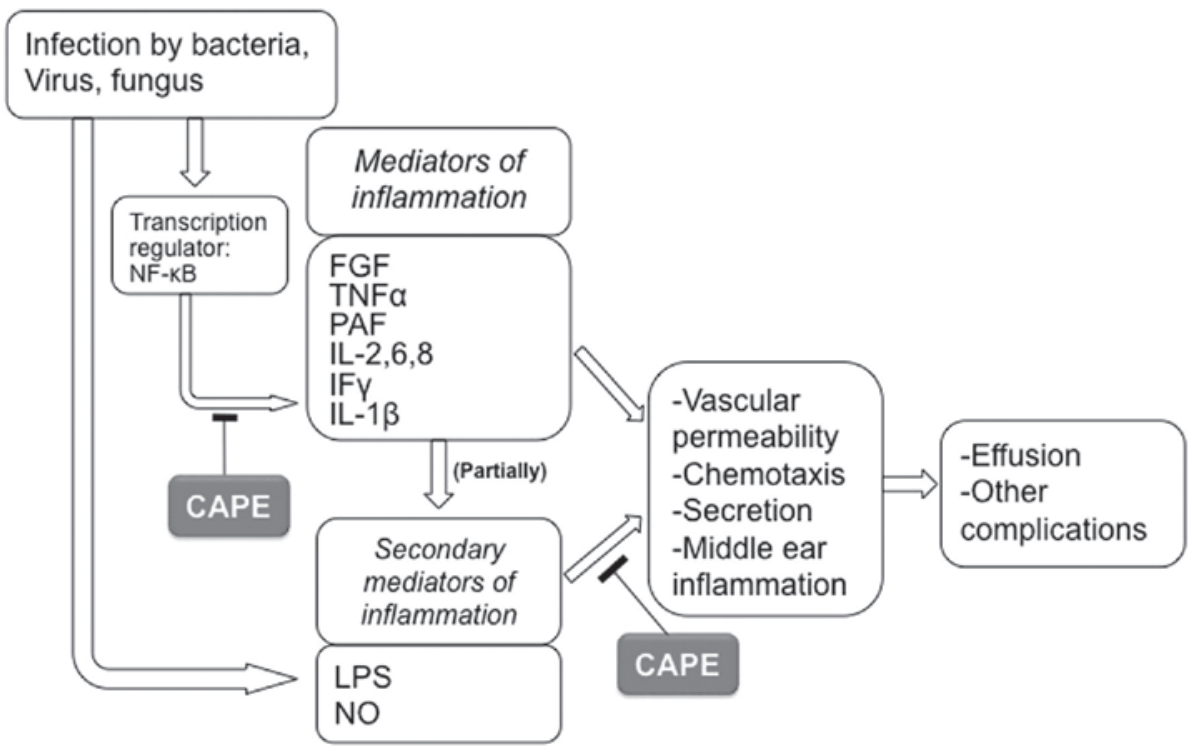

Figure 4. Proposed mechanism by which CAPE diminishes or blocks otitis media via different pathways. The transcription regulator, NF-kB has been shown to be involved in regulating the expression of large numbers of genes, such as cytokines, chemokines and other mediators involved in inflammatory responses. CAPE is a potent and specific inhibitor of NF- $\mathrm{KB}$. FGF, fibroblast growth factor; PAF, platelet activating factor; LPS, lipopolysaccharide; NO, nitric oxide; CAPE, caffeic acid phenethyl ester; IL, interleukin; NF-kB, nuclear factor-kB; TNF- $\alpha$, tumor necrosis factor $\alpha$.

suppressed LPS-induced TNF- $\alpha$ expression, IL-8 production and NF- $\mathrm{kB}$ activity in HMEEC cells, according to the results of quantitative polymerase chain reaction (qPCR), enzyme-linked immunosorbent assay (ELISA) and western blot analyses.

Previous reports have demonstrated that CAPE is an inhibitor of NF- $\mathrm{\kappa B}$, which is a regulatory molecule that modifies the expression of genes associated with cell proliferation, inflammatory responses and cell adhesion. Downregulation of several pro-inflammatory cytokines is caused by the inhibition of NF- $\mathrm{kB}$ (29). Ordinarily, NF- $\kappa B$ is inactive in the cytoplasm,

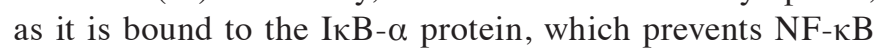
activity. When inflammation occurs, I $\kappa \mathrm{B}-\alpha$ is phosphorylated and degraded, which results in the translocation of NF- $\kappa B$ into the nucleus, where it activates the expression of a number of genes associated with inflammatory responses. Therefore, the inhibition of I $\kappa \mathrm{B}-\alpha$ phosphorylation may prevent NF- $\kappa \mathrm{B}$ translocation (30). LPS treatment induces the degradation of IкB- $\alpha$. CAPE treatment results in the inhibition of LPS-induced I $\kappa$ B- $\alpha$ degradation in a dose-dependent manner, which prevents inflammation. Overall, the literature provides an insight into the molecular pathways underlying the anti-inflammatory effects of CAPE in association with OM and other inflammatory conditions.

\section{Use of CAPE in hydrogen peroxide $\left(\mathrm{H}_{2} \mathrm{O}_{2}\right)$-induced oxidative stress in HMEEC cells}

Recently, Song et al examined the inhibitory effects of CAPE on $\mathrm{H}_{2} \mathrm{O}_{2}$-induced oxidative stress in HMEEC cells (31). $\mathrm{H}_{2} \mathrm{O}_{2}$ was added at different concentrations for up to $6 \mathrm{~h}$. The $\mathrm{H}_{2} \mathrm{O}_{2}$-induced inflammatory response was subsequently analyzed by quantifying TNF- $\alpha$ and COX-2 mRNA expression, using reverse transcription-quantitative PCR. CAPE was applied at different concentrations for 30 and $60 \mathrm{~min}$, following which, ROS production, including superoxide dismutase (SOD) expression, was determined. $\mathrm{H}_{2} \mathrm{O}_{2}$ caused an increase in SOD protein expression. However, treatment of the cells with CAPE inhibited the expression of the SOD protein. The authors observed that CAPE treatment decreased $\mathrm{H}_{2} \mathrm{O}_{2}$-induced ROS production in HMEEC cells. Additionally, CAPE treatment inhibited COX-2 protein and TNF- $\alpha$ mRNA expression, which were upregulated following treatment with $\mathrm{H}_{2} \mathrm{O}_{2}$ alone. Therefore, CAPE may inhibit $\mathrm{H}_{2} \mathrm{O}_{2}$-induced oxidative injury and reduce the expression of inflammatory mediators, which are involved in the pathophysiology of OM.

\section{Effect of CAPE in myringosclerosis (MS)}

MS is described as hyalinization and calcification in the collagen layer of the tympanic membrane. Histopathological examination indicates an increase in collagen fibers, as well as in extracellular calcium deposition and hyaline degeneration in the lamina propria (32). Although the exact mechanisms underlying the development of MS are unknown, ROS and related oxidative stress are among the possible causes of this condition (33). Following placement of tubes into the tympanic membranes, hyperoxia occurs, which results in the excessive production of ROS (34). The preventive effects of CAPE on the development of MS in tympanic membranes of myringotomized rats have been investigated, using otomicroscopy and histopathology (35). Following myringotomy, rats were treated with CAPE for two weeks. Otomicroscopic evaluation was performed and extensive myringosclerotic plaques were observed. However, the plaques observed in myringotomized rats treated with CAPE were less extensive, compared with those treated with saline solution. Similarly, histopathological evaluation revealed extensive sclerotic membranes in the tympanic membranes of non-treated blank control as well as in saline-treated groups. Sclerotic deposits and fibroblast infiltration/production were observed in the lamina propria. 
Thickening of the tympanic membranes was also observed. The tympanic membranes of the myringotomized rats that had been subjected to CAPE treatment were thinner compared with those in the group without CAPE treatment. Furthermore, the degree of fibroblastic activity in the lamina propria was lower in CAPE-treated myringotomized rats compared with those that were not treated with CAPE. In conclusion, the preventive effects of CAPE on the development of MS in myringotomized rats appears to be associated with a number of mechanisms, including the scavenging activity of free radicals.

\section{Conclusions}

This review discusses only a small number of in vivo studies on ear disease, and the use of CAPE exceeds the scope of this article. In addition to its use in a number of in vivo studies of ear pathology, as an antioxidant and anti-inflammatory agent, beneficial effects of CAPE have been reported in a number of types of cancer, arthritis, allergy, heart disease, diabetes, kidney disease, liver disease and neurological disease. CAPE modulates a number of biochemical pathways and several targets involved in ear diseases, such as ototoxicity. CAPE treatment has demonstrated promising effects in oxidative stress-mediated and inflammation-mediated diseases (Fig. 2). CAPE may be a useful treatment for patients with ear disease. Further investigation of the effects of different doses of CAPE, as well as appropriate administration regimens and the bioavailability of this agent in humans is required.

\section{References}

1. Koltuksuz U, Ozen S, Uz E, et al: Caffeic acid phenethyl ester prevents intestinal reperfusion injury in rats. J Pediatr Surg 34: 1458-1462, 1999

2. Ilhan A, Koltuksuz U, Ozen S, Uz E, Ciralik H and Akyol O: The effects of caffeic acid phenethyl ester (CAPE) on spinal cord ischemia/reperfusion injury in rabbits. Eur J Cardiothorac Surg 16: 458-463, 1999.

3. Ozyurt H, Söğüt S, Yildirim Z, et al: Inhibitory effect of caffeic acid phenethyl ester on bleomycine-induced lung fibrosis in rats. Clin Chim Acta 339: 65-75, 2004.

4. Iraz M, Ozerol E, Gulec M, et al: Protective effect of caffeic acid phenethyl ester (CAPE) administration on cisplatin-induced oxidative damage to liver in rat. Cell Biochem Funct 24: 357-361, 2006.

5. Orban Z, Mitsiades N, Burke TR Jr, Tsokos M and Chrousos GP: Caffeic acid phenethyl ester induces leukocyte apoptosis, modulates nuclear factor-kappa B and suppresses acute inflammation. Neuroimmunomodulation 7: 99-105, 2000.

6. Natarajan K, Singh S, Burke TR Jr, Grunberger D and Aggarwal BB: Caffeic acid phenethyl ester is a potent and specific inhibitor of activation of nuclear transcription factor NF-kappaB. Proc Natl Acad Sci USA 93: 9090-9095, 1996.

7. Huang Q and Tang J: Age-related hearing loss or presbycusis. Eur Arch Otorhinolaryngol 267: 1179-1191, 2010.

8. Sha SH, Taylor R, Forge A and Schacht J: Differential vulnerability of basal and apical hair cells is based on intrinsic susceptibility to free radicals. Hear Res 155: 1-8, 2001.

9. Akyol S, Ozturk G, Ginis Z, Armutcu F, Yigitoglu MR and Akyol O: In vivo and in vitro antineoplastic actions of caffeic acid phenethyl ester (CAPE): Therapeutic perspectives. Nutr Cancer 65: 515-526, 2013.

10. Ozturk G, Ginis Z, Akyol S, Erden G, Gurel A and Akyol O: The anticancer mechanism of caffeic acid phenethyl ester (CAPE): Review of melanomas, lung and prostate cancers. Eur Rev Med Pharmacol Sci 16: 2064-2068, 2012.

11. Akyol S, Ginis Z, Armutcu F, Ozturk G, Yigitoglu MR and Akyol O: The potential usage of caffeic acid phenethyl ester (CAPE) against chemotherapy-induced and radiotherapy-induced toxicity. Cell Biochem Funct 30: 438-443, 2012.
12. Khalil MI and Sulaiman SA: The potential role of honey and its polyphenols in preventing heart diseases: a review. Afr J Tradit Complement Altern Med 7: 315-321, 2010.

13. Kizilay A, Kalcioglu MT, Ozerol E, et al: Caffeic acid phenethyl ester ameliorated ototoxicity induced by cisplatin in rats. J Chemother 16: 381-387, 2004.

14. Rizzi MD and Hirose K: Aminoglycoside ototoxicity. Curr Opin Otolaryngol Head Neck Surg 15: 352-357, 2007.

15. Poirrier AL, Pincemail J, Van Den Ackerveken P, Lefebvre PP and Malgrange B: Oxidative stress in the cochlea: An update. Curr Med Chem 17: 3591-3604, 2010.

16. Sanli A, Aydin S, Sarisoy ZA, Paksoy M, Ayduran E and Erdivanli OC: The protective effect of dexamethasone and lactate against cisplatin-induced ototoxicity. Turk J Med Sci 41: 467-474, 2011.

17. van den Berg JH, Beijnen JH, Balm AJ and Schellens JH: Future opportunities in preventing cisplatin induced ototoxicity. Cancer Treat Rev 32: 390-397, 2006.

18. Yorgason JG, Luxford W and Kalinec F: In vitro and in vivo models of drug ototoxicity: Studying the mechanisms of a clinical problem. Expert Opin Drug Metab Toxicol 7: 1521-1534, 2011.

19. Rybak LP and Whitworth CA: Ototoxicity: Therapeutic opportunities. Drug Discov Today 10: 1313-1321, 2005.

20. Bakir S, Ozbay M, Gun R, et al: The protective role of caffeic acid phenethyl ester against streptomycin ototoxicity. Am J Otolaryngol 34: 16-21, 2013.

21. Sakallioglu O, Yalcin S, Ozel HB, Colakoglu N and Alpay HC: Prenatally exposure to exogenous glucocorticoids and stress may affect the inner ear. Kulak Burun Bogaz Ihtis Derg 23: 104-111, 2013.

22. BarrettTQ,Kristiansen LH andOvesen T: NF-kappaB incultivated middle ear epithelium. Int J Pediatr Otorhinolaryngol 67: 895-903, 2003.

23. Yellon RF, Leonard G, Marucha PT, et al: Characterization of cytokines present in middle ear effusions. Laryngoscope 101: 165-169, 1991.

24. Rose AS, Prazma J, Randell SH, Baggett HC, Lane AP and Pillsbury HC: Nitric oxide mediates mucin secretion in endotoxin-induced otitis media with effusion. Otolaryngol Head Neck Surg 116: 308-316, 1997.

25. Li W, Lin J, Adams GL and Juhn SK: Expression of inducible nitric oxide synthase (iNOS) in middle ear epithelial cells by IL-1beta and TNF-alpha. Int J Pediatr Otorhinolaryngol 55: 91-98, 2000.

26. Watanabe K, Tomiyama S, Jinnouchi K, Pawankar R and Yagi T: Expression of inducible nitric oxide synthase in the cochlea following immune response in the endolymphatic sac of guinea pigs. ORL J Otorhinolaryngol Relat Spec 63: 155-159, 2001.

27. Wright DT, Fischer BM, Li C, Rochelle LG, Akley NJ and Adler KB: Oxidant stress stimulates mucin secretion and PLC in airway epithelium via a nitric oxide-dependent mechanism. Am J Physiol 271: L854-L861, 1996.

28. Song JJ, Cho JG, Hwang SJ, Cho CG, Park SW and Chae SW: Inhibitory effect of caffeic acid phenethyl ester (CAPE) on LPS-induced inflammation of human middle ear epithelial cells. Acta Otolaryngol 128: 1303-1307, 2008.

29. Shin KM, Kim IT, Park YM, et al: Anti-inflammatory effect of caffeic acid methyl ester and its mode of action through the inhibition of prostaglandin E2, nitric oxide and tumor necrosis factor-alpha production. Biochem Pharmacol 68: 2327-2336, 2004.

30. Kopp EB and Ghosh S: NF-kappaB and rel proteins in innate immunity. Adv Immunol 58: 1-27, 1995.

31. Song JJ, Lim HW, Kim K, Kim KM, Cho S and Chae SW: Effect of caffeic acid phenethyl ester (CAPE) on $\mathrm{H}_{2} \mathrm{O}_{2}$ induced oxidative and inflammatory responses in human middle ear epithelial cells. Int J Pediatr Otorhinolaryngol 76: 675-679, 2012.

32. Mattsson C, Marklund SL and Hellstrom S: Application of oxygen free radical scavengers to diminish the occurrence of myringosclerosis. Ann Otol Rhinol Laryngol 106: 513-518, 1997.

33. Mattsson $\mathrm{C}$ and Hellstrom S: Inhibition of the development of myringosclerosis by local administration of fenspiride, an anti-inflammatory drug. Eur Arch Otorhinolaryngol 254: 425-429, 1997.

34. Felding JU, Rasmussen JB and Lildholdt T: Gas composition of the normal and the ventilated middle ear cavity. Scand J Clin Lab Invest Suppl 186: 31-41, 1987.

35. Song JJ, Kwon SK, Cho CG and Park SW: The effect of caffeic acid phenethyl ester on the prevention of experimentally induced myringosclerosis. Int J Pediatr Otorhinolaryngol 71: 1287-1291, 2007. 